\title{
Let the Anti-Drug Awareness Go Deep into the Youth: On the Innovative Path of Drug Control Publicity in Colleges and Universities
}

\author{
Jun Yin ${ }^{1}$, Jiaxin Liao ${ }^{1}$, Xiaona Luo ${ }^{2 *}$, Xi Cheng ${ }^{3}$ \\ ${ }^{1}$ Guangdong University of Foreign Studies South China Business College, Zhongluotan Town, Baiyun District, \\ Guangzhou, China \\ ${ }^{2}$ School of English Language and Culture, Guangdong University of Foreign Studies, North Baiyun Avenue, Huangshi \\ Street, Baiyun District, Guangzhou, China \\ ${ }^{3}$ School of Automotive Engineering, Jilin University, Nanling Subdistrict, Nanguan District, Changchun, China \\ *Corresponding author. Email: 805836006@qq.com
}

\begin{abstract}
Drugs are the root of all evils. Since the eradication of tobacco in Humen nearly 200 years ago, the Chinese nation has never stopped fighting against drugs. Anti-drug work has always been a problem that our Party and government attach great importance to, and anti-drug publicity and education is a key measure in drug control. From the perspective of the history of drug control, the publicity of drug control has gradually developed into mass media from oral propaganda, billboards, slogans and brochures. As an important position in drug control, colleges and universities must implement drug control propaganda based on their own, so that every college student understands the importance of drug control. In addition, the role of college students should be changed from passive input of education to active publicity, that is, to take the form of youth spokespersons to conduct anti-drug publicity through new media. In this regard, this paper puts forward the innovative path of anti-drug publicity based on the actual situation of anti-drug publicity in colleges and universities, so as to promote the development of anti-drug cause.
\end{abstract}

Keywords: anti-drug propaganda, college students, educational measures, innovation

\section{INTRODUCTION}

In recent years, the people's war on drugs has achieved new results as the nation has intensified its efforts to crack down on drugs. At present, China's drug crimes, especially new drug crimes, show an integrated development trend of drug production, drug trafficking and consumption ${ }^{[1]}$, and the cases of cross-border production, trafficking and smuggling of new drugs are increasing sharply. In spite of the continuous promotion of drug prevention and education projects for teenagers in China, the number of teenagers who take drugs still occupies a relatively high proportion. Especially with the continuous development of the Internet era, college students become the focus of criminals. The number of college students participating in drug-related activities through the Internet is increasing day by day ${ }^{[2]}$. However, due to the influence of factors such as the social psychology of college students and the increase of economic burden, it also lays hidden dangers for drug taking caused by indirect contact with drugs. As the main position of anti-drug prevention education, colleges and universities play an important role.

General Secretary Xi Jinping pointed out that "domestic and overseas drug problems, the harm of traditional and new drugs, and online and offline drug crimes are intertwined, causing serious harm to people's life safety and health and social stability. We must continue to resolutely and thoroughly carry out anti-drug work as always." The propaganda and education of adolescent drug control is an important link in the national drug control strategy, and also one of the fronts in the war on drug control. Therefore, to do a good job of anti-drug publicity and education in colleges and universities can help the war on drugs, which is of great significance to the education of college students. Therefore, colleges and universities must make use of their own advantages, do a good job in the propaganda and education of drug control, strengthen their guard against drugs and sense of responsibility for drug control, guide college students to reject drugs from the source, and kill drugs in the cradle. 


\section{PROBLEMS OF ANTI-DRUG PROPAGANDA IN COLLEGES AND UNIVERSITIES}

\subsection{Common Problems}

By analyzing the results of the questionnaire sampling survey conducted by Wei Chunsheng and Wang Dan on college students by means of self-design, visit, correspondence survey and network survey ${ }^{[1]}$, we can find that college students lack drug knowledge; also, there are some common problems in the anti-drug publicity and education work of colleges and universities in China, such as inadequate recognition, old content, and lack of teachers; most importantly, college students are not deep enough in drug knowledge study, which leads to three characteristics of their drug awareness weak cognition, low vigilance and no defensiveness ${ }^{[3]}$.

\subsection{Three Characteristics of College Students' Perception of Drugs}

\subsubsection{Weak Cognition}

According to the current understanding of drugs among college students, some students have weak cognition of drugs and have unclear or incomplete understanding of the harm of drug addiction. Drugs are not only harmful to the human body, but also easy to increase the risk of HIV infection. Students' weak cognition leads to problems in anti-drug propaganda.

\subsubsection{Low Vigilance}

College students, in the late youth and early growth stage, tend to produce a variety of psychological contradictions. They are in this process of rapid physical and psychological change, thus easy to use drugs and other sexual stimulants for self-relief ${ }^{[4]}$.

\subsubsection{No Defensiveness}

College students do not have a certain understanding of drugs, and their safety awareness is relatively low. Without any precautions, there will naturally be danger.

\subsection{Other Problems}

\subsubsection{Anti-drug Publicity Units with Inadequate Cooperation and Incomplete System}

According to the current anti-drug propaganda mode in colleges and universities, there is no comprehensive anti-drug education system of "government-society-school-family" based on the actual situation of colleges and universities. As a result, there are all kinds of problems in publicity, especially the lack of the coordination mechanism between school and family, school and government, which is not conducive to the overall development.

\subsubsection{Overly Formalized Anti-drug Propaganda}

In the process of drug control, the form of propaganda is very simple and formalized, without in-depth understanding of the actual situation of college students. Anti-drug propaganda pays too much attention to the publicity of common drugs, such as heroin, methamphetamine, ecstasy, ketamine, etc. There is little mention of new drugs, such as laughing gas, GHB and Mdmb-chmica, which become the target of many teenagers' drug use. In addition, lectures are usually used in the publicity process, which are boring and less interactive, leading to students' lack of interest in anti-drug publicity, which makes anti-drug propaganda and education unable to play its due effect.

\subsubsection{Low Participation of College Students in Anti-drug Propaganda}

The main time for anti-drug publicity is around the International Day against Drug Abuse and Illicit Trafficking on June 26 every year. The long-term publicity is relatively few, so that students cannot truly understand the harmfulness of drugs and the importance of anti-drug. The insufficient atmosphere of anti-drug propaganda in schools leads to the low participation of students in the process of anti-drug propaganda. Especially in the blank period of anti-drug publicity, students do not understand the knowledge of anti-drug, which makes the previous publicity in vain. With low participation of students, the effect of anti-drug propaganda will naturally decrease ${ }^{[5]}$.

\section{EFFECTIVE MEASURES FOR ANTI-DRUG PUBLICITY IN COLLEGES AND UNIVERSITIES}

\subsection{Give Play to the Role of Local Anti-drug Committees in the Publicity of Colleges and Universities}

General Secretary Xi Jinping pointed out that "party committees and governments at all levels should adhere to the people-centered development philosophy. With a highly responsible attitude towards the country, the nation, the people and history, we will adhere to the policy of strict drug control and fight the people's war on drugs well. We will improve the drug control system, deepen international cooperation in drug control, and make continuous progress in drug control. We will make new and greater contributions to maintaining social harmony and stability and ensuring that the people live and work in peace and contentment." Therefore, the local drug control committee must combine with the actual 
situation and play their role in the anti-drug publicity of colleges and universities. For example, around November every year, before the final examination week, college students have both energy and time to learn anti-drug knowledge. Local anti-drug commissioners could send staff to local colleges and universities to preach, namely anti-drug lectures. Every year in May, before the International Day against Drug Abuse and the final examination week of college students, is good for publicity. Local schools can organize students to visit the nearby anti-drug museum, so that students can learn anti-drug knowledge firsthand.

At the same time, in the future development, the drug control committee should combine the development trend of The times and adopt the integrated communication mode of "short video plus anti-drug publicity", that is, using the integrated thinking of Internet and media to actively guide and encourage other communication subjects to produce and disseminate short anti-drug videos. Combined with the actual situation of college students, now there are not only anti-drug micro film, anti-drug public service announcements, anti-drug music videos, and anti-drug animation, but also appeared rap, videos of flashing text, mini-drama and other forms of expression. These diversified forms of communication are very close to the fashion trend of college students ${ }^{[6]}$.

\subsection{Combine Theory with Practice and Let Anti-drug Knowledge Enter the Heart}

In the actual development, colleges and universities should take advantage of their own resources and policy advantages to actively connect with communities and society to establish drug-free schools and hold drug-free community activities, so as to lay a foundation for the development of drug control. Colleges and universities should pay attention to expanding educational ideas and combine drug control work with conventional management. According to students' cognitive characteristics, various anti-drug education activities should be carried out to help students understand the harm of drugs and the basic skills of drug prevention and anti-drug policies and regulations, and form a positive and healthy way of life and study ${ }^{[7]}$. For example, carry out situational simulation content design to guide students to apply anti-drug knowledge into real life; organize knowledge competitions to consolidate students' knowledge; conduct student volunteer propaganda to spread anti-drug knowledge to other students by student volunteers. At the same time, in the name of universities and the local anti-drug committee and other relevant units to jointly carry out anti-drug publicity, so as to improve the understanding of teachers and students on anti-drug. It is worth noting that the social practice during the holidays is also a very good platform, that is, to carry out a research activity with students as the main body during the holidays, which helps to widely publicize drugs and strengthen students' understanding of drugs.

In addition, colleges and universities should strengthen the cooperation with the community, and guide college students to understand the livelihood of the community, so as to improve the social cognition of college students. Local governments can carry out grid management based on the actual situation, so as to realize the refinement of community management and provide institutional support for drug control. College student volunteers can cooperate with the community to do a good job in anti-drug publicity. In a word, only by participating in it can college students deepen their understanding of drug control and lay a foundation for their future development.

\subsection{Youth Spokesmen: Striving to Be Anti-drug Ambassadors on Campus}

It is worth noting that in the process of drug prevention, college students are not only the object of drug prevention education, but also the transmission of drug prevention, as well as a very important volunteer team of drug prevention. China has a large number of college graduates every year. If these people have received scientific drug prevention education, it will achieve an unpredictable positive transmission power. Thus we must combine the actual situation of colleges and universities to let more college students participate in it ${ }^{[8]}$. Let the youth speak, that is, encourage college students to become anti-drug ambassadors on campus. The role of student union changes from passive education to active publicity, which strengthens college students' understanding of drugs and promotes their sense of social responsibility. At the same time, in the actual development, we should pay attention to making reasonable anti-drug decisions and constructing good campus culture guidance, so as to give full play to the role of anti-drug propaganda ${ }^{[9]}$.

\section{CONCLUSION}

Life is only once, so the young should cherish it more. Don't let "I thought" become "I regret"! In short, taking college students as the carrier to do a good job in anti-drug publicity is an inevitable trend of The times. Anti-drug publicity needs to establish correct communication ideas, realize the combination of anti-drug publicity and college students' endorsement, and give play to the advantages of anti-drug publicity in the new era.

\section{Project Fund:}

[1] Investigation and Countermeasures of Unconventional Love Views under the Dual Model of Community Support and Peer Counseling Based on EAP 
Concept, General projects, social investigation reports and academic papers in philosophy and social sciences, pdjh2021B0686

[2] Strategy Discussion on The Establishment of Campus Recruitment Market Practice -- Based on the Creation of university Student Entity Enterprise Southern Talent Market, General projects, social investigation reports and academic papers in philosophy and social sciences, pdjh2019b0654

\section{REFERENCES}

[1] Wang Yinhua, Shi Jingxuan, Li Puchu. Current Situation and Innovation Path of Anti-drug Propaganda and Education in Colleges and Universities. Journal of Baoding University. 201,34(03).

[2] Xi Jinping Made Important Instructions on Drug Control, Stressing Adherence to the Policy of Strictly Enforcing Drug Control in Order to Fight the People's War on Drug Control and Promote New Achievements in Drug Control Work [N]. People's Daily, 2020-06-24(1).

[3] Wei Chunsheng, Wang Dan. Investigation and Analysis of Anti-drug Propaganda and Education in Colleges and Universities in China. Chinese Journal of Drug Dependence. 2010.19(01).

[4] Wang Lige. New Challenges and Countermeasures of Drug Prevention Education in Colleges and Universities in the Post-Epidemic Era Based on the Use of New Media. Journal of Yunnan Police Officer Academy. 2021,(03).

[5] Wang Jianping. Investigation and Research on Youth Anti-drug Publicity and Education in Guangzhou from the Perspective of Ecosystem [J]. Journal of Guangzhou City Polytechnic, 201,15(03):67-72.

[6] Mo Guanyao, Luo Tingyin. Innovative Path Exploration of "Cultural Drug Prohibition" -Taking Foshan Practice as an Example [J]. Journal of Yunnan Police Officer Academy, 2021(04):14-18.

[7] Wang Yinhua, Shi Jingxuan, Li Puchu. Current Situation and Innovation Path of Anti-drug Publicity and Education in Colleges and Universities [J]. Journal of Baoding University, 201,34(03):111-116.

[8] Xi Zhiwu, Xue Yuanyuan. Research on Fusion Communication of "Short Video plus Anti-drug Propaganda" [J]. Journal of Guangxi Police College, 201,34(02):107-114.

[9] Wang Xue, Li Wenjun, Chen Shuaifeng. Evaluation on Drug-related Cognition and Education Effect of
College Students in Beijing [J]. Chinese Journal of School Health, 201,42(02):215-219. 